\title{
Diabetes mellitus in der stationären Versorgung von Patienten mit Migrationshintergrund
}

\author{
Besonderheiten bei diesen Patienten
}

\author{
Elisabeth Wesselman', Bodo Gutt ${ }^{2}$ \\ Fachreferat für Interkulturelle Versorgung \\ 2 Abteilung für Endokrinologie, Diabetologie, Suchtmedizin, Nuklearmedizin, Klinikum München GmbH; Klinikum Schwabing; München
}

In Deutschland leben mehr als 15 Millionen Menschen mit Migrationshintergrund [1]. „Migration kann einen Einfluss haben sowohl auf das Risiko zu erkranken als auch auf die Chance, eine adäquate Therapie zu erhalten. Spezifische Risiken für Migranten können sich aus dem Minoritätenstatus ergeben, aus einem, verglichen mit der Mehrheitsbevölkerung, schlechteren Zugang zur Gesundheitsversorgung (zum Beispiel aufgrund sprachlicher oder kultureller Barrieren) sowie aus den möglichen psychosozialen Belastungen der Migration“ [2]. Vor allem ältere Migranten treffen im Gesundheitswesen auf informationsbedingte, kulturelle und kommunikative Barrieren [3], die zu bekannten Problemen von Unter-, Über- und Fehlversorgung bei Therapie und Pflege führen. „Einerseits werden Hausärzte häufiger als von Deutschen aufgesucht, Notfallambulanzen der Krankenhäuser häufiger in Anspruch genommen, erfolgen Einweisungen ins Krankenhaus häufiger, ist die Verweildauer höher und werden mehr Medikamente verordnet. Andererseits sind Migrantinnen in der fachärztlichen Versorgung - insbesondere bei Psychiaterinnen und Neurologinnen - sowie in Präventions- und Rehabilitationsmaßnahmen deutlich unterrepräsentiert" [4].

Epidemiologische Studien, die den Migrationsstatus miterfassen und Aussagen zur Prävalenz von ausgewählten Erkrankungen machen können, sind kaum zu finden. Die aktuelle Datenlage zur Beschreibung des Gesundheitszustandes von Migrantinnen und Migranten ist als nicht ausreichend anzusehen. In der gesundheitswissenschaftlichen Forschung „liegen - mit Ausnahme der ersten Ergebnisse des Kinder- und Jugendgesundheitssurveys (KiGGS) [5] - aktuell keine repräsentativen Untersuchungen zur gesundheitlichen Situation von Migranten vor, noch wurde diese Zielgruppe in der Vergangenheit ausdrücklich und in sinnvoller Differenziertheit in Studien über die Wohnbevölkerung von Deutschland einbezogen“ [6]. Viele der in Deutschland verfügbaren Routinedaten unterscheiden nicht
In Deutschland leben mehr als 15 Millionen Menschen mit Migrationshintergrund. Vor allem ältere Migranten treffen im Gesundheitswesen auf informationsbedingte, kulturelle und kommunikative Barrieren, die zu Problemen von Unter-, Überund Fehlversorgung führen. Um eine adäquate medizinische Versorgung für diese Patientengruppe zu ermöglichen, wurden die anonymisierten Patientendaten des Klinikinformationssystems (SAP-ISH) am Städtischen Klinikum München (StKM) retrospektiv für das Kollektiv der Patienten mit Diabetes mellitus (DM) ausgewertet. Von 2007 bis 2009 hat das StKM an 5 Standorten insgesamt über 515000 Patienten versorgt, davon 51329 (9,97\%) nichtdeutscher Nationalität. 9420 Patienten wurden mit der Hauptdiagnose DM behandelt, 1016 von diesen mit nichtdeutscher Staatsangehörigkeit. Der Anteil Nichtdeutscher stieg im Beobachtungszeitraum kontinuierlich von 8,7 auf $12,5 \%$ an. Die am häufigsten dokumentierten nichtdeutschen Nationalitäten der Patienten mit der Hauptdiagnose DM waren dabei türkisch (34,2\%), ex-jugoslawisch $(22,4 \%)$ und griechisch $(6,8 \%)$. $26,8 \%$ der 8407 deutschen Patienten wurden wegen einer Typ-1-Erkrankung behandelt, 68,8\% wegen einer Typ-2-Erkrankung. Die Kohorte der nichtdeutschen Patienten unterschied sich dabei nicht signifikant von der deutschen Kohorte (27,3 und 69,2\%). Allerdings fiel in den Subgruppen der türkischen und griechischen Patienten ein vermehrtes Auftreten von Typ-2-Erkrankungen auf (81,8 und 79,7\%). Während sich deutsche Patienten mit der Hauptdiagnose DM im Mittel mit 59,1 Jahren stationär vorstellten, wurden nichtdeutsche Patienten 7,8 Jahre früher mit einem mittleren Alter von 51,3 Jahren stationär aufgenommen. Die Daten von 1016 Patienten nichtdeutscher Nationalität aus München und Umgebung zeigen, dass diese Patientenklientel absolut wie relativ zunimmt sowie jünger hospitalisiert wird. Diese bedeutsame Personengruppe muss in der klinischen Forschung eine besondere Berücksichtigung erfahren, um migrationsspezifische Versorgungsprobleme aufdecken zu können.

ausreichend zwischen Menschen mit und ohne Migrationshintergrund [7]. In dem Bericht des Robert Koch Instituts zu Migration und Gesundheit wird dieser Mangel durch: „ungelöste technische und interpretatorische Probleme, Mange an belastbaren Daten zur Deckung des vorhanden Informationsbedarfs“ begründet [8]. Gezielte Untersuchungen vorhandener Daten aus dem Krankenhausinformationssystem - wie die hier vorliegenden - können zur Urs forschung beitragen, bestehende Ungleich in der Versorgung aufdecken und eine bessere Einbindung von Migranten in Präventionsprogramme unterstützen. 


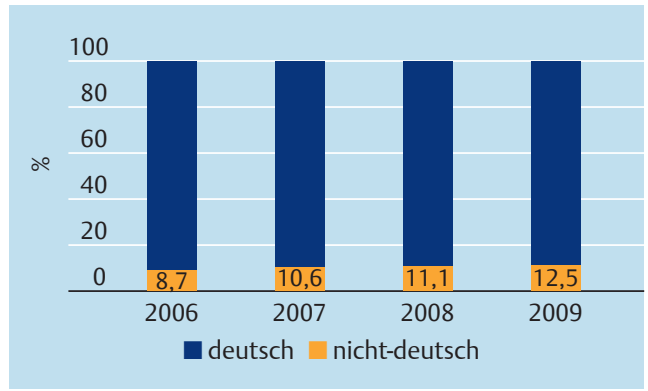

Abb. 1 Im Verlauf der Behandlungsjahre 2006 bis 2009 kam es neben der kontinuierlichen Zunahme aller Patienten mit der Hauptdiagnose Diabetes mellitus (im Mittel 2\% pro Jahr) zu einem überproportionalen Anstieg der nichtdeutschen Patienten (von 8,7\% in 2006 auf $12,5 \%$ in 2009 ).

\section{Material und Methode}

In dieser retrospektiv angelegten Untersuchung wurden die in der Datenbank des Patienteninformationssystems SAP-ISH (SAP GmbH) digital erfassten Patientendaten der Jahrgänge 2006 bis 2009 anonymisiert ausgewertet. Mittels Datenbankabfragen wurden die Parameter Alter, Geschlecht, Nationalität und Hauptdiagnose als ICD-10-Schlüssel gewonnen. Zur Auswertung der rekrutierten Daten hinsichtlich der Gewinnung von Aussagen zum Diabetes mellitus schränkten wir die Datenbankabfrage auf Datensätze mit der zum Diabetes mellitus gehörigen Hauptdiagnose E10.xx bis E14.xx ein. Medizintouristen und Patienten unter 18 Jahre wurden aus der Auswertung ausgeschlossen.

Das Städtische Klinikum München (StKM), einer der größten Gesundheitsdienstleister in der Landeshauptstadt München, hat die kultursensible Versorgung von Migranten/innen als ein wichtiges Qualitätsziel festgelegt. Deshalb wird seit 2006 bei der Klinikaufnahme auf freiwilliger Basis die Staatsangehörigkeit aller Patienten erhoben und in der Software zur Patientenadministration mit erfasst.

Die Staatsangehörigkeit aller Patienten der ehemaligen Sozialistischen Föderativen Republik Jugoslawien bzw. Bundesrepublik Jugoslawien wurde in der Klinikdatenbank bis 2008 als eine Gruppe „Jugoslawien“ geführt. Ab 2009 erfolgte die getrennte Erfassung der Staatsangehörigkeiten Bosnien-Herzogowina, Kosovo, Kroatien, Montenegro, Mazedonien, Serbien und Slowenien im Klinikinformationssystem. Um jedoch eine statistische Vergleichbarkeit im longitudinalen Verlauf zu ermöglichen, mussten letztere gemeinsam als Gruppe „ex-Jugoslawien“ ausgewertet werden.

\section{Statistische Auswertung}

Für die statistische Analyse wurde das Programm StatView (SAS Institute Inc., USA) verwandt. Alle erhobenen Parameter werden, soweit nicht

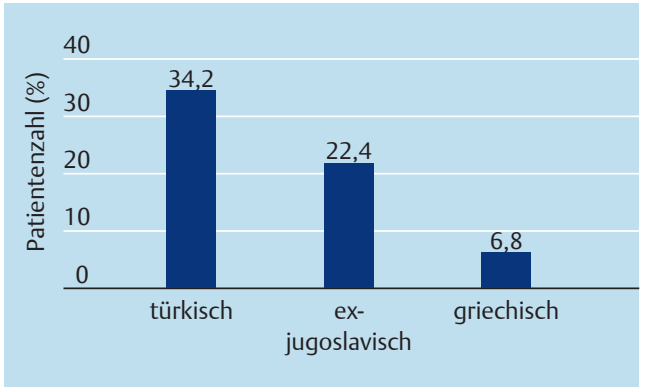

Abb. 21016 der 9499 Patienten mit einer Hauptdiagnose zum Diabetes mellitus (ICD E10.xx bis E14.xx) waren im Beobachtungszeitraum 2006 bis 2009 nichtdeutscher Staatsangehörigkeit. Die TOP-3-Nationalitäten nichtdeutscher Patienten waren: türkisch (34,2\%), ex-jugoslawisch $(22,4 \%)$ und griechisch $(6,8 \%)$.

anders bezeichnet, als Mittelwert (und Standardabweichung) angegeben. Zur statistischen Auswertung der normalverteilten Kohorte wurden parametrische Testverfahren (t-Test) verwandt. Als Signifikanztest auf Unabhängigkeit in der Kontingenztafel diente der Exakte FisherTest. Das Signifikanzniveau wurde mit einem pWert $<0,05$ als signifikant, mit einem $\mathrm{p}$-Wert $<0,01$ als hochsignifikant festgelegt.

\section{Ergebnisse}

Im Untersuchungszeitraum von 2006 bis 2009 hat das StKM an 5 Standorten insgesamt über 515000 Patienten versorgt. Von diesen gaben 51329 Patienten eine nichtdeutsche Nationalität an $(9,97 \%)$.

9420 Patienten wurden mit der Hauptdiagnose Diabetes mellitus stationär behandelt. Davon waren 5372 (57\%) Männer und 4048 (43\%) Frauen. 1016 der 9420 Patienten (10,8\%) gaben eine nichtdeutsche Staatsangehörigkeit an.

Im Verlauf der Behandlungsjahre 2006 bis 2009 konnten wir eine kontinuierliche Fallzahlzunahme bei Patienten mit der Hauptdiagnose Diabetes mellitus (im Mittel 2\% pro Jahr) unabhängig von ihrer Staatsangehörigkeit aufzeigen. Demgegenüber konnten wir einen überproportionalen Fallzahlanstieg in der Subgruppe der nichtdeutschen Patienten ermitteln. So war in 2006 der Anteil nichtdeutscher Patienten in der Kohorte der Patienten mit Diabetes mellitus 8,7\%, 2007 10,6\%, 2008 11,1\% und 2009 12,5\% (Abb. 1).

Die 1016 nichtdeutschen Patienten mit Diabetes mellitus rekrutierten sich aus 66 Nationen. Die 3 häufigsten Nationalitäten waren türkisch (34,2\%), ex-jugoslawisch (22,4\%) und griechisch (6,8\%). Mit 642 von 1016 Fällen stellten diese 3 Nationalitäten $63,4 \%$ des nichtdeutschen Kollektivs (Abb. 2).

Im Beobachtungszeitraum stellten sich 2532 Patienten mit Typ-1-Diabetes und 6487 mit Typ-2Diabetes als Hauptdiagnose stationär vor. Wäh- 


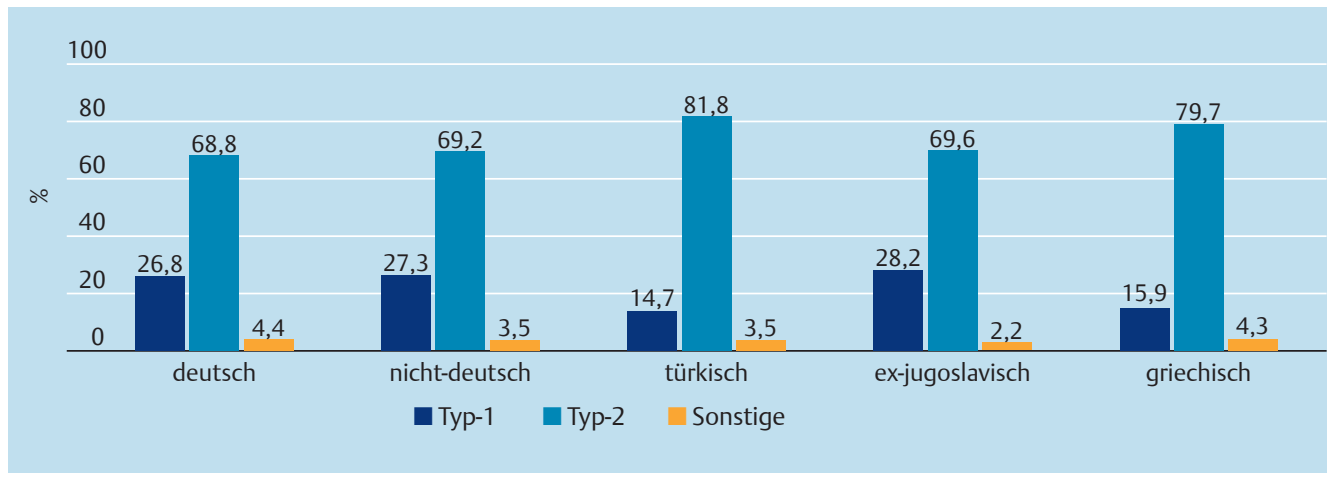

Abb. 3 Häufigkeitsverteilung der Subgruppen der Patienten mit Typ-1-, Typ-2-Diabetes und sonstigen Diabetesformen: während sich die Verteilung der Diabetestypen zwischen deutschen und der Gesamtheit der nichtdeutschen Patienten nicht signifikant unterscheidet, ist in der Subgruppe der türkischen $(p<0,0001)$ und griechischen $(p<0,05)$ Patienten eine signifikante Überhäufigkeit der Typ-2-Erkrankung zu registrieren.

rend sich die Verteilung der Diabetestypen zwischen deutschen und der Gesamtheit der nichtdeutschen Patienten nicht signifikant unterscheidet (Typ-1-Erkrankte: 26,8 bzw. 27,3\%; Typ-2-Erkrankte: 68,8 bzw. 69,2\%; $p=0,851$ ), war in der Subgruppe der türkischen $(\mathrm{p}<0,001)$ und griechischen Patienten $(\mathrm{p}<0,05)$ eine prozentuale Überhäufigkeit der Typ-2-Erkrankung im Vergleich zur deutschen Kohorte zu registrieren (Typ-1-Erkrankte: 14,7 bzw. 15,9\%; Typ-2Erkrankte: 81,8 bzw. 79,7\%; Abb. 3).
Das mittlere Alter bei der stationären Aufnahme war 58,2 Jahre ( $S D \pm 22,2$ Jahre). Das Alter der 4048 Frauen war mit 57,70 Jahren $( \pm 20,07$ Jahren) signifikant jünger als das der Männer (58,96 $\pm 24,79$ Jahre; $p=0,0062)$. Die Patienten mit Typ-1-Diabetes stellten sich im Mittel mit 33,8 Jahren ( $\pm 21,0$ Jahren), die mit Typ-2-Diabetes mit 68,1 Jahren ( $\pm 13,3$ Jahren; $\mathrm{p}<0,0001)$ vor.

Nichtdeutsche Patienten mit Diabetes mellitus stellten sich mit einem mittleren Alter von 51,29 


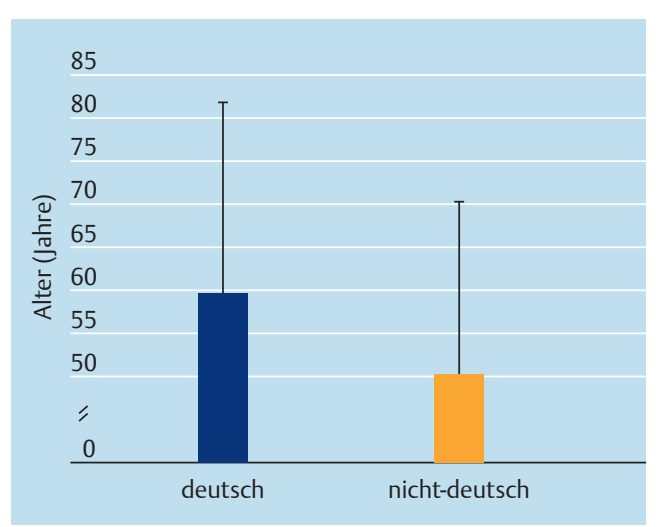

Abb. 4 Während sich deutsche Patienten mit der Hauptdiagnose Diabetes mellitus im Mittel mit 59,1 Jahren stationär vorstellten, wurden nichtdeutsche Patienten 7,8 Jahre früher, mit einem mittleren Alter von 51,3 Jahren, stationär aufgenommen.

Jahren ( $\pm 19,60$ Jahre) um 7,8 Jahre früher vor als deutsche Patienten $(59,08 \pm 22,38$ Jahre, $\mathrm{p}<0,0001 ;$ Abb. 4).

\section{Diskussion}

Im Bereich der Gesundheitsberichterstattung wird einerseits der Mangel an validen Daten zum Gesundheitszustand von Migranten beklagt, der gerade für die Versorgungsforschung von hoher Bedeutung sei, um Menschen beim Erhalt ihrer Gesundheit zu unterstützen, andererseits darauf hingewiesen, dass das Merkmal „Staatsangehörigkeit“" keine befriedigende Auskunft geben würde, da z.B. Aussiedler, Spätaussiedler, Menschen mit erworbener deutscher Staatsangehörigkeit nicht erfasst würden [7]. Gewarnt wird vor einer eingeschränkten Sichtweise, die Krankheiten der „Migration“ zuschreibt, statt auch soziale und ökonomische Faktoren ausreichend $\mathrm{zu}$ berücksichtigen.

Ein deutlicher Zusammenhang zwischen Migration und Krankheit wird jedoch bei der Verbreitung, Ausprägung und dem Verlauf chronischer Erkrankungen wie bei Diabetes mellitus gesehen [9].

Allerdings ist die Aussagekraft bisheriger öffentlich zugänglicher Daten unbefriedigend. Weder Krankenkassen noch Arztpraxen oder Krankenhäuser erfassen in der Regel Patientenmerkmale hinsichtlich Migration.

In einer Publikation des bundesweiten „Arbeitskreis Migration und öffentliche Gesundheit“ wurde im Rahmen eines Fachtages zur migrationssensiblen Gesundheits- und Pflegeberichterstattung die Krankenhausdiagnosestatistik als Datenquelle für die kommunale Gesundheitsberichterstattung untersucht und aufgrund der Erfassung der Diagnose, Behandlung, Alter und des Geschlechts von Patienten als geeignet bewertet [10]. Kritisiert wird der Mangel an
Patientenmerkmalen hinsichtlich der sozialen Lage und des Migrationshintergrundes. In der gleichen Publikation wird berichtet, wie einige wenige Institutionen der öffentlichen Gesundheitsversorgung - wie z.B. das Städtische StKM München $\mathrm{GmbH}$ - versuchen diesen Mangel in ihrer Datenerfassung zu beheben. Mit der Erhebung der Staatsangehörigkeit will das Klinikum Kenntnisse darüber gewinnen, welche medizinisch-pflegerischen Leistungen von Migrantinnen und Migranten in Anspruch genommen werden, welche Muttersprache sie vermutlich sprechen und welche Unterstützungsangebote sie eventuell benötigen könnten.

Darüber hinaus kann die große Kohorte von Patienten gleicher Diagnose Mitarbeitern der Klinik gezielte Untersuchungen - wie die hier vorliegende - zur Versorgungsforschung ermöglichen, um bestehende Ungleichheiten in der Versorgung aufzudecken und eine bessere Einbindung von Migranten in Behandlungsund Präventionsprogramme zu unterstützen [8].

Die gewonnen Ergebnisse zeigen, dass die Inanspruchnahme von stationärer Behandlung aufgrund eines Diabetes mellitus durch nichtdeutsche Patienten stetig zunimmt. Erklären lässt sich dies durch das Alter, das die erste Generation der Migranten erreicht hat. Viele von ihnen kamen als Gastarbeiter in den 1960er Jahren und bleiben zunehmend als Rentner in Deutschland. Mehr als die Hälfte der Migranten stammten dabei aus dem Mittelmeerraum, davon 1,9 Mio. Türken. In Bayern besitzen die meisten Migranten die Staatsbürgerschaft eines anderen europäischen Landes (40\%), der Türkei (20,3\%) oder von Staaten des ehemaligen Jugoslawiens (17,5\%) [11]. Dieses Verhältnis spiegelt sich auch bei der Zusammensetzung unserer untersuchten Patienten wider.

Ohne dies genau nachweisen zu können, sprechen auch Fachgesellschaften von einem zu erwartenden Anstieg: „Erste Untersuchungen weisen darauf hin, dass die Diabeteshäufigkeit unter Migranten höher ist als unter der deutschen Ursprungsbevölkerung. Mit zunehmendem Lebensalter steigt die Wahrscheinlichkeit, an Diabetes mellitus zu erkranken. Durch die Alterung der ersten Migrantengeneration wird sich deshalb die Zahl der ausländischen Diabetiker schon in den nächsten Jahren verdoppeln.“ [12]. Diese postulierte Annahme stützen auch unsere Daten.

Das zeigt, dass sich Gesundheitseinrichtungen wie Arztpraxen und Krankenhäuser darauf einrichten müssen, zukünftig vermehrt mit der Versorgung dieser Patienten zu tun zu haben. Die Deutsche Diabetes Gesellschaft gibt auf ihrer Homepage 600000 Diabetiker mit Migrationshintergrund an und weist in diesem Zusammenhang auf die 2001 
verabschiedete „Bad Neuenahrer Erklärung“ hin, die bestehende Barrieren und Hemmnisse in der Versorgung insbesondere mit sprachlichen Verständigungsschwierigkeiten, unterschiedlichen Essgewohnheiten sowie einem anderen kulturellen und religiösen Hintergrund sowie einem traditionellen Verständnis von Krankenrolle und Therapiezielen erklärt [12].

Außer einem kontinuierlichen Anstieg der Diabetiker-Patienten nichtdeutscher Nationalität zeigt unsere Auswertung, dass bisherige Studien, die meist auf die Untersuchung von türkischen Mitbürgern beschränkt sind, nicht das gesamte Ausmaß an Behandlungs- und/oder Präventionsdefiziten von Migranten erfassen.

Türkische Migranten sollen durchschnittlich 10 Jahre früher als Nichtmigranten an schweren chronischen Krankheiten erkranken [13]. Bekannt sind Studien aus Gießen, in der bei Türken bei dem Check-Up-35 eine Prävalenz des Diabetes von über $8 \%$, in einer Bevölkerungsstudie in Frankfurt sogar von fast 15\% gefunden wurde [14]. Diese Problematik der chronischen Erkrankung Diabetes mellitus betrifft nicht nur Menschen türkischer Herkunft. Insbesondere zur Erfassung des Krankheitsstandes anderer Nationalitäten müssen vermehrte Anstrengungen gemacht werden.
Das bei den Migrantengruppen durchgängig ermittelte niedrigere Durchschnittsalter bei klinischer Inanspruchnahme kann sicher nicht nur damit erklärt werden, dass bei den „alten“ Migranten nicht, wie vergleichsweise bei den deutschen Patienten, Hochaltrigkeit zu finden ist, da deren Aufenthalt in der Bundesrepublik zu kurz bzw. ein Teil der Migranten im Alter wieder in das Herkunftsland zurückgekehrt ist. Es stellt sich hier die Frage, ob Migranten früher eine klinische Behandlung bei Diabetes mellitus benötigen, weil sie vergleichsweise früher und/oder schwerer als deutsche Mitbürger erkranken. Hier wäre sicher das in der Literatur häufig genannte defizitäre Gesundheitsverhalten zu nennen [15], dass bei Patienten mit ungünstigen sozialen Bedingungen häufig zu finden ist. Es könnten aber auch spezifische Behandlungsdefizite, eine immer noch ineffiziente Prävention, wesentliche Ursache sein. Diese Frage lässt sich durch die Auswertung unserer Krankenhausdiagnosestatistik nicht klären.

Hier stellt sich die Notwendigkeit weiterer Untersuchungen. Es muss u.a. geprüft werden, inwieweit auch andere Parameter des metabolischen Syndroms - wie Übergewicht - herangezogen werden müssen $[8,11]$.

Zusammenfassend lassen die Auswertung der Daten des Städtischen Klinikums München 
Diabetes Mellitus in the Inpatient Management of Patients with a Migration Background - Special Features of these Patients

In Germany there are more than 15 million people with a migration background. Older immigrants in particular are confronted with information-related, cultural on communication barriers that can lead to the problems of under- or over management or even the wrong management. In order to enable an adequate medical care for this patient group the anonymized patient data of the clinic information system (SAP-ISH) at the Städtischen Klinikum München (StKM) were retrospectively evaluated for patients with diabetes mellitus (DM). From 2007 to 2009 the StKM treated a total of over 515,000 patients at 5 locations, of these 51,329 (9.97\%) were of non-German nationalities. 9420 patients were treated for the main diagnosis DM, 1016 of these were of nonGerman nationalities. The proportion of non-Germany increased continuously in the observation period from $8.7 \%$ to $12.5 \%$. The most frequently documented non-German nationalities of patients with the primary diagnosis DM were Turkish (34.2\%), former Yugoslavian (22.4\%) und Greek (6.8\%). 26.8\% of the 8407 German patients were treated for type 1 disease, $68.8 \%$ for type 2 disease. The cohort of non-German patients did not differ significantly from the German cohort in this respect (27.3\% und 69.2\%). However, in the subgroup of Turkish and Greek patients an increased occurrence of type 2 disease was apparent ( 81.8 und $79.7 \%$ ). Whereas the German patients presented with the primary diagnosis DM at an average age of 59.1 years, the non-German patients presented for hospitalization 7.8 years earlier with an average age of 51.3 years. The data of 1016 patients of non-German nationalities from Munich and surroundings show that these patient groups both absolutely and relatively are increasing in size and are admitted to hospital at younger ages. This significant group of people should receive particular attention in clinical research in order to be able to uncover migration-specific health-care problems.

Key words

diabetes mellitus, chronic disease, migration, immigration, intercultural

\section{Danksagung: Wir danken} Achim März und Roland Wimmer (Controlling Datenverarbeitung/Städtischen Klinikum München $\mathrm{GmbH}$ ) für die Unterstützung bei der Datenbankabfrage und Datenrekrutierung.

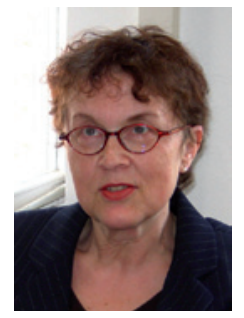

Korrespondenz

Elisabeth Wesselman

Fachreferat für

Interkulturelle Versorgung

Städtisches Klinikum

München $\mathrm{GmbH}$

Krankenhaus Schwabing

Kölner Platz 1

80803 München

E-Mail: elisabeth.wesselman@

klinikum-muenchen.de

\section{Autorenerklärung}

Die Autoren erklären, dass für diesen Artikel keine

Interessenkonflikte bestehen.
GmbH zu der Erkrankung von Menschen anderer Nationalität mit Diabetes mellitus folgende Aussagen zu:

1. Die Inanspruchnahme klinischer Behandlung von Diabetes mellitus steigt im Untersuchungszeitraum kontinuierlich.

2. Der im Vergleich zu den deutschen Patienten höhere prozentuale Anteil einer Typ-2-Erkrankung bei türkischen und griechischen Patienten zeigt, dass die in bisherigen Studien durchgeführte Fokussierung auf die Gruppe der Patienten türkischer Herkunft vermutlich nur einen Teil der bestehenden Probleme aufzeigt. Untersuchungen müssen gezielt alle Migrantengruppen unterschiedlicher Nationalität umfassen.

3. Das im Vergleich zu Deutschen ermittelte jüngere Durchschnittsalter bei klinischer Inanspruchnahme deutet auf eine eventuelle frühere Erkrankung von Migranten an Diabetes mellitus oder eine häufigere Diabeteserkrankung von Migranten hin, kann aber auch im wesentlichen Versorgungsmängel (Behandlung, Prävention) zur Ursache haben. Hier fehlen weitere Untersuchungen, z.B. Kenntnisse zu dem Alter, bei der die Erstdiagnose gestellt wurde.
4. Offen ist ebenfalls, ob die im Städtischen Klinikum München $\mathrm{GmbH}$ behandelten Migranten mit einem Typ-2-Diabetes aufgrund von Versorgungsmängel mit einer vergleichsweise leichten Erkrankung, die in der Regel nicht krankenhauspflichtig ist, stationär behandelt wurden, oder ob aufgrund vom Schweregrad eine stationäre Behandlung unbedingt erforderlich war. Auch hier müssen weitere Untersuchungen folgen.

5. Die vorliegenden Daten erlauben die Schlussfolgerung, dass Migranten mit Diabetes mellitus eine konsequente sowie besonders personalisierte und aufgrund des vermutlich niedrigeren Durchschnittalters eine langfristig gesicherte, nebenwirkungsarme Therapie benötigen.

\section{Literatur}

1 Loeffelholz HD. Update Migration - aktuelle Grunddaten der Zuwanderung nach Deutschland. Vortrag im Rahmen der 11. Jahrestagung der Akademie Öffentliches Gesundheitswesen Düsseldorf: Migration - Herausforderung für das öffentliche Gesundheitswesen. Düsseldorf 28.10.2010

2 Razum O, Geiger I, Zeeb H. Ronellenfitsch U. Gesundheitsversorgung von Migranten. Dtsch Arztebl 2004; 101: A 2882-A2887

3 Pott E. Gesundheitliche Chancengleichheit für Migrantinnen und Migranten. Strategien und Handlungsempfehlungen der BZgA im Bereich Migration und Gesundheit. Bundeszentrale für gesundheitliche Aufklärung (BZgA). Vortrag im Rahmen der 11. Jahrestagung der Akademie Öffentliches Gesundheitswesen Düsseldorf: Migration - Herausforderung für das öffentliche Gesundheitswesen. Düsseldorf 28.10.2010

4 Palecek F. Ältere Migrantinnen - Soziale Lage und Gesundheit. In: Migration \& Gesundheit. Dossier Heinrich-Böll-Stiftung. Berlin. 2009: 22-25

5 Beauftragte der Bundesregierung für Migration, Flüchtlinge und Integration. 7. Bericht über die Lage der Ausländerinnen und Ausländer in Deutschland. Berlin. 2007

6 Butler J, Albrecht N-J, Ellsäßer G et al. Migrationssensible Datenerhebung für die Gesundheitsberichterstattung. Bundesgesundheitsbl-GesundheitsforschGesundheitsschutz 2007; 50: 1232-1239

7 Razum O, Spallek J. Wie gesund sind Migranten? Erkenntnisse und Zusammenhänge am Beispiel der Zuwanderer in Deutschland. Bundeszentrale für politische Bildung, focus migration Kurzdossier Nr. 12. 2009

8. Razum $\mathrm{O}$, Zeeb $\mathrm{H}$, Meesmann $U$ et al. Ulrich $\mathrm{R}$. Migration und Gesundheit. Schwerpunktbericht der Gesundheitberichterstattung des Bundes Robert Koch Institut Berlin 2008

9 Knipper M, Bilgin Y. Migration und Gesundheit. Konrad Adenauer Stiftung Berlin 2009

10 Migrationssensible Datenerhebung für die Gesundheits- und Pflegeberichterstattung. Dokumentation, Fachkonferenz 21.11.2008 Berlin

11 Gesundheit und Migration. Bayerischer Bericht. Gesundheitsberichterstattung für Bayern 4. Bayerisches Landesamt für Gesundheit und Lebensmittelsicherheit. Erlangen 2011

12 www-deutsche-diabetes-gesellschaft.de

13 Ulusoy N, Grässel E. Turkish emigrants in Germany. Deficits in home care knowledge and health services - a review. Z Gerontol Geriatr 2010

14 Icks A, Kulzer B, Razum O. Diabetes bei Migranten. In: Deutscher Gesundheitsbericht Diabetes 2011

15 Brenner G, Altenhofen L, Knoepnadel J, Weber I. Nationale Gesundheitsziele: Diabetes mellitus Typ 2 als Zielbereich Bundesgesundheitsbl. Gesundheitsforsch Gesundheitsschutz 2003; 46: 134-143 